\title{
Preface to the volume Languages: Bioinspired Approaches
}

\author{
Gemma Bel-Enguix
}

Languages, whether they be natural or artificial, are particular cases of a symbol system. And the manipulation of symbols is the stem of formal language theory. The theory of formal languages mainly originated from mathematics and generative linguistics. It was born in the middle of the 20th century as a tool for modelling and investigating the syntax of natural languages. After 1964, it developed as a separate branch with specific problems, techniques and results and since then it has had an important role in the field of computer science. Formal language theory, due to its abstract and formal properties, has been applied to a wide range of fields (besides initial linguistic motivation): economic modelling, developmental biology, cryptography, sociology... Therefore, natural languages, computer science and formal languages had a mutual influence over the years.

During the 20th century, biology became a pilot science, and many disciplines have formulated their theories using models taken from nature. Bio-informatics, bio-engineering and bio-linguistics are good examples of this pre-eminence of 'bio'-sciences. Computer science has almost become another bio-inspired field thanks to the considerable development of natural computing and DNA computing.

Following this tradition of interaction between biology and computer science, the articles in this volume present bioinspired approaches to formal language therory. Artion Alhazov and his co-author Tseren-Onolt Ishdorj 
develop some aspects of Membrane Computing in the first two papers of the journal. Membranes are also the central topic of Mihai Ionescu's contribution, that introduces P Systems with promoted/inhibited rules and traces. Adria-Horia Dediu presents some applications of evolutionary algorithms to formal languages. María Dolores Jiménez-López applies one of the main processes in DNA, recombination, to formalize some patterns in syntax of natural languages. The paper by Remco Loos and Benedek Nagy explains some concepts of parallelism in DNA computing. Finally, the contribution by K.G. Subramanian, Mary Roslin and P. Helen Chandra is focused in the description of splicing for bidimensional languages.

The work that is published in this volume has been been previously presented and discussed in the weekly seminars that the GRLMC organized during several years. The authors have improved and updated articles that provide new ideas, tools and formalisms which can help to the description, analyis and processing of natural and artificial languages.

I am deeply grateful to all the contributors, for their professional and friendly cooperation, and to the Universitat Rovira i Virgili, for supporting the publication of this issue. 